\title{
Superconductivity in intercalated buckled two-dimensional materials: $\mathrm{KGe}_{2}$
}

\author{
Sherif Abdulkader Tawfik, ${ }^{1,2, \text { | }}$ Catherine Stampfl, ${ }^{3}$ and Michael J. Ford ${ }^{1, \dagger}$ \\ ${ }^{1}$ School of Mathematical and Physical Sciences, University of Technology Sydney, Ultimo, New South Wales 2007, Australia. \\ ${ }^{2}$ Institute for Biomedical Materials and Devices (IBMD), Faculty of Science, \\ University of Technology Sydney, Sydney, NSW, Australia. \\ ${ }^{3}$ School of Physics, The University of Sydney, Sydney, New South Wales, 2006, Australia.
}

\begin{abstract}
Germanene has emerged as a novel two-dimensional material with various interesting properties and applications. Here we report the possibility of superconductivity in a stable potassium intercalated germanene compound, $\mathrm{KGe}_{2}$, with a transition temperature $T_{c} \sim 11 \mathrm{~K}$, and an electron-phonon coupling of 1.9. Applying a 5\% tensile strain, which reduces the buckling height by $4.5 \%$, leads to the reduction of the electron-phonon coupling by $11 \%$ and a slight increase in $T_{c} \sim 12 \mathrm{~K}$. That is, strong electron-phonon coupling results from the buckled structure of the germanene layers. Despite being an intercalated van der Waals material similar to intercalated graphite superconductors, it does not possess an occupied interlayer state.
\end{abstract}

\section{INTRODUCTION}

The largest class of high-temperature superconductors includes materials that are formed of intercalated twodimensional (2D) structures, such as $\mathrm{YBa}_{2} \mathrm{Cu}_{3} \mathrm{O}_{7}$ and iron-based superconductors. The simplest such structure is $\mathrm{MgB}_{2}$, in which a layered flat hexagonal material is intercalated by a single element, which was discovered in $2001^{1]}$ to have a high $T_{c}$ of $39 \mathrm{~K}$. In 2005 , another class of intercalated 2D materials was found to superconduct: graphite intercalated with $\mathrm{Ca}$ and $\mathrm{Yb} ! 2$ This class of structures, known as graphite intercalated compounds (GIC), is fundamentally different to $\mathrm{MgB}_{2}$, because it is formed of graphene layers that can exist individually as a stable crystal. Since 2005, the number of new GICs has not increased; to date, the only known superconducting GICs are those with intercalatants K, Ca, Li, Yb, Sr and Ba. This discovery created immense research interest aiming to understand the mechanism that underlies the superconductivity in this class of materials.

In spite of the structural similarity of GICs to $\mathrm{MgB}_{2}$, the superconducting temperature of $\mathrm{MgB}_{2}$ is far higher than all of the observed $T_{c}$ 's of GICs, the maximum on record being $11.5 \mathrm{~K}$ for $\mathrm{CaC}_{6} \cdot{ }^{2]}$ Moreover, it was observed that a superconducting gap appears on the Fermi surface associated with the intercalatant atom, but not in the $\pi^{*}$ orbital of the graphitic layers, ${ }^{3}$ confirming the theoretical prediction that there is an occupied interlayer state in all superconducting compounds of this class of materials. 4 While $\mathrm{MgB}_{2}$ possesses an interlayer state, it is unoccupied. Another key difference is the fact that $\mathrm{MgB}_{2}$ is a two-gap superconductor ${ }^{1}$ and the two gaps were theoretically shown $n^{5}$ to arise from the $\sigma$ and $\pi$ bands. However, graphite intercalated compounds are single gap superconductors.

Nevertheless, graphite intercalation compounds and $\mathrm{MgB}_{2}$ have a number of features in common. The hexagonal 2D layers in both are perfectly flat; that is, there is no buckling. In fact, buckling was thought to destroy the superconducting state in layered $\mathrm{Li}_{x} \mathrm{BC}$ because it induces strong mixing of the $\sigma$ and $\pi$ bands ${ }^{[6]}$ The cal- culated electron-phonon coupling (EPC) strength, $\lambda$, of bulk $\mathrm{CaC}_{6}$ is $0.83,^{7}$ and that of $\mathrm{MgB}_{2}$ is $0.748^{5}$, so the EPC strength in both compounds are quite close.

It is interesting to consider what would then be the superconducting properties of an intercalated vdW material that is intrinsically buckled? An example of a buckled 2D material is germanene, which can be viewed as the cleaved (111) layer of the $F d 3 m$ phase of bulk germanium. It is predicted to be a stable 2D Dirac material ${ }^{8}$ Here we explore the potential superconductivity of potassium-intercalated germanene, $\mathrm{KGe}_{2}$, which is a hypothetical compound that resembles the structure of $\mathrm{CaGe}_{2}$ that is already well known! ${ }^{9}$ The interesting feature of $\mathrm{KGe}_{2}$ is that the germanene layers preserve their Dirac cones; that is, $\mathrm{KGe}_{2}$ is a truly intercalated $2 \mathrm{D}$ germanene material. The $\mathrm{K}$ intercalation was also observed to enhance the superconductivity of FeSe, and the $\mathrm{K}$ intercalation of $\mathrm{MoS}_{2}$ leads to the emergence of several superconducting phases. 11 We find by solving the anisotropic Eliashberg equations based on densityfunctional theory (DFT) and time-dependent perturbation theory ${ }^{15}$ that the superconducting gap is $\sim 11 \mathrm{~K}$, which is close to that observed in $\mathrm{CaC}_{6}$.

\section{COMPUTATIONAL DETAILS}

The DFT calculations are performed using the local density approximation (LDA) $)^{\sqrt{12}}$ and norm-conserving pseudopotentials 13 using QUANTUMESPRESSO14] The valence electronic wave functions are expanded in a plane-wave basis set with a kinetic energy cutoff of 40 Ry. We use a $12 \times 12 \times 12 \mathrm{k}$-point mesh for $\mathrm{KGe}_{2}$ and $12 \times 12 \times 1$ for monolayer germanene, and a MethfesselPaxton smearing $\frac{15}{15}$ of $0.10 \mathrm{eV}$. The dynamical matrices and the linear variation of the self-consistent potential are calculated within density-functional perturbation theory $\sqrt{16}$ on the irreducible set of a regular $12 \times 12 \times 12$ k-point mesh and a $4 \times 4 \times 4$ q-point mesh for $\mathrm{KGe}_{2}$, and $12 \times 12 \times 1$ k-point mesh and a $4 \times 4 \times 1$ q-point mesh for germanene. In order to solve the Eliashberg equations we evaluate electron energies, phonon frequencies, and 
electron-phonon matrix elements on fine grids with a $N_{k}=20 \times 20 \times 20$ Monkhorst-Pack mesh and a $N_{q}=20 \times 20 \times 20$ for $\mathrm{KGe}_{2}$ and $N_{k}=20 \times 20 \times 1$ Monkhorst-Pack mesh and a $N_{q}=20 \times 20 \times 1$ for germanene, which were obtained by convergence of the EPC. The calculations are performed using smearing parameters in the Dirac $\delta$ functions corresponding to 100 and $0.5 \mathrm{meV}$ for electrons and phonons, respectively.

The EPC, $\lambda$, is calculated according to the equation

$$
\lambda=2 \int \frac{\alpha^{2} F(\omega)}{\omega} d \omega .
$$

where the Eliashberg spectral function $\alpha^{2} F(\omega)$ is defined as

$$
\alpha^{2} F(\omega)=\frac{1}{2 \pi N\left(e_{F}\right)} \sum_{\mathbf{q} \nu} \delta\left(\omega-\omega_{\mathbf{q} \nu}\right) \frac{\gamma_{\mathbf{q} \nu}}{\hbar \omega_{\mathbf{q} \nu}}
$$

where $\omega$ is the phonon frequency, $\mathbf{q}$ and $\nu$ are the phonon momentum and mode, respectively, $N\left(e_{F}\right)$ is the electronic density of states at the Fermi level, and $\gamma_{\mathbf{q} \nu}$ the electron-phonon coupling strength associated with a specific phonon mode $\nu$ and momentum $\mathbf{q}$.

\section{RESULTS AND DISCUSSIONS}

In all our calculations of the layered $\mathrm{KGe}_{2}$ we have used the $A A$ stacking of the germanene layers as displayed in Fig. 1. The $A A$ stacking was found to be more favorable than the $A B$ stacking based on comparing the stabilization energies of the two configurations, $E_{S}$, which is defined as $E_{S}=E_{K G e_{2}}-E_{G e_{2}}-E_{K}$, where $E_{K G e_{2}}$ is the total energy of $\mathrm{KGe}_{2}, E_{\mathrm{Ge}_{2}}$ is the total energy of the germanene unit cell and $E_{K}$ is the total energy of an isolated $\mathrm{K}$ atom. The $E_{S}$ of $A A$ was calculated to be $-2.46 \mathrm{eV}$, which is $126 \mathrm{meV}$ lower than that of $A B$. Finding the optimal stacking sequence for intercalated layered compounds is a daunting task, we choose here to only study the $A A$ stacking based on the following considerations: This unit cell is the smallest of all $\mathrm{KGe}_{2}$ structures, which offers computational efficiency. Even though other stacking sequences are possible, the $A A$ stacking is a good representative model of the layered $\mathrm{KGe}_{2}$ for the purpose of calculating the superconducting properties, because the small horizontal shifts in the position of the $\mathrm{K}$ atoms across the layers are expected to have little effect on its superconducting properties ${ }^{17}$. The calculated lattice parameters of the $A A \mathrm{KGe}_{2}$ structure are $a=3.987 \AA$ And $c=4.596 \AA$, while that of monolayer germanene is $a=3.921 \AA$. The buckling height of the germanene layers in $\mathrm{KGe}_{2}$ (the $z$-axis distance between the two Ge atoms) is $0.827 \AA$, while that of monolayer germanene is $0.620 \AA$, in agreement with published results $\frac{8}{8}$ With such a crystal, the K-K bond length becomes 3.987 $\AA$, which is higher than the bond length of the K-K bond $(3.577 \AA)$ in the $\operatorname{Im} 3 m \mathrm{~K}$ polymorph at $12 \mathrm{GPa}{ }^{[22}$

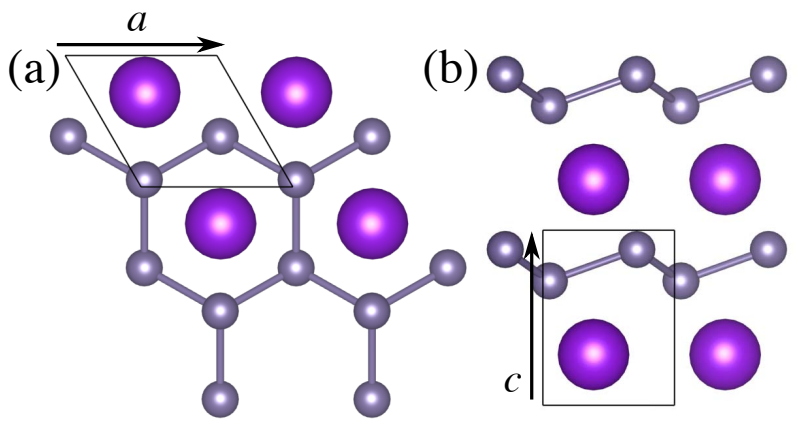

FIG. 1: The (a) top and (b) side view of the $\mathrm{KGe}_{2}$ structure with the $A A$ stacking. The unit cell is indicated with associated lattice vectors. The $\mathrm{K}$ atom is positioned in the center of the germanene hole.

In order to examine the impact of buckling on the EPC and the superconducting properties, we applied a planar strain on $\mathrm{KGe}_{2}$. Specifically, we tested the strains along the $a$ axis of $\pm 5 \%$ and $\pm 10 \%$. We found that $-5 \%$ and $-10 \%$ tensile strains result in dynamically unstable structures (that is, the phonon dispersion has imaginary frequencies). In the case of positive strains, a $5 \%$ strain (with the lattice parameter $a=4.212 \AA$ ) maintains the dynamical stability of the structure, whereas $10 \%$ strain yields a dynamically unstable structure. Therefore, we focus here on the structure with $5 \%$ positive strain. The buckling height in this structure is $0.790 \AA$, which is $4.5 \%$ less than that of the unstrained structure.

With the buckling of the germanene layers in $\mathrm{KGe}_{2}$, the coupling between the $\pi$ band and the $\mathrm{K}$ band is expected to be much stronger than in $\mathrm{MgB}_{2}$ and $\mathrm{CaC}_{6}$. The band structure of $\mathrm{KGe}_{2}$ and the projected density of states are presented in Fig. 2(a,b), and the band structure of monolayer germanene is diplayed in Fig. 2(c). In the unit cell, according to Bader charge analysis, the $\mathrm{K}$ atom donates $1|e|$ to the germanene layer, which results in shifting the Dirac point of germanene in Fig. 2(b) downwards. This full charge transfer is analogous to the nearly-full charge transfer that takes place in $\mathrm{KC}_{8} \sqrt{19}$.

As the Dirac cone is shifted below the Fermi level, there is one Ge $4 p$ band, that is slightly hybridized with $\mathrm{K} 3 p$, crossing the Fermi energy. Compared to the $\mathrm{CaC}_{6}$ band structure, the Dirac cone in $\mathrm{KGe}_{2}$ stays intact, while in $\mathrm{CaC}_{6}$ the $\Gamma$ point is opened by $\sim 0.5 \mathrm{eV}$. The Dirac cones here do not experience a momentum shift, unlike the situation in $\mathrm{CaSi}_{2}$ (which is non-superconducting) where the Dirac cone shifts slightly from the high symmetry points of the first Brillouin zone due to the electron transfer between the adjacent silicene layers ${ }^{18}$. The situtation of $\mathrm{KGe}_{2}$ is akin to $\mathrm{KC}_{8}{ }^{19}$ (a superconductor), and electrostatically doped graphene, where only minor differences related to the intercalation states are present close to Fermi energy at the $\Gamma$ point.

It is of interest to determine whether $\mathrm{KGe}_{2}$ exhibits an interlayer state like intercalated graphite superconductors? The presence of such a state in superconduct- 


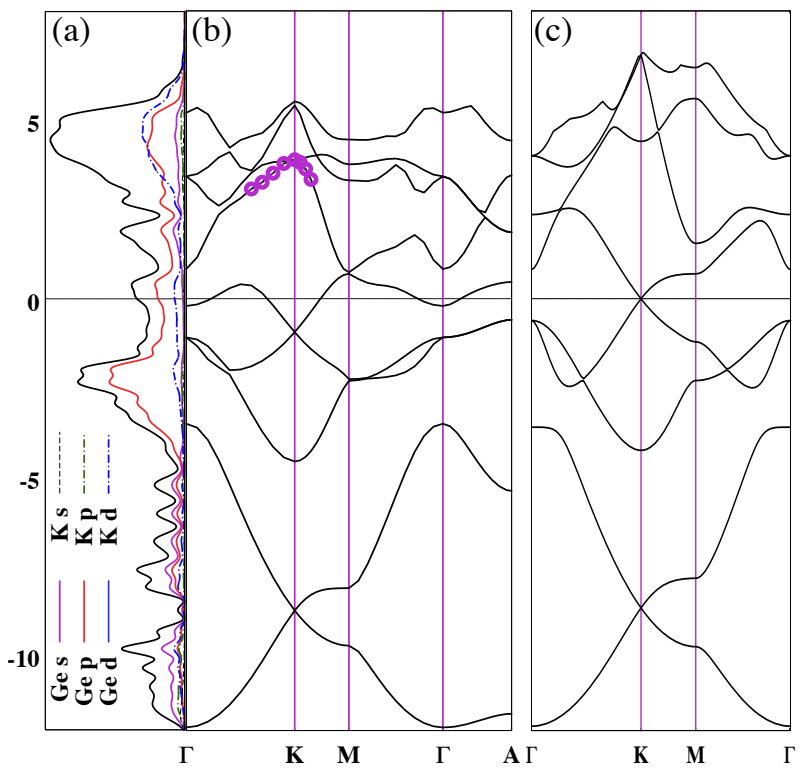

FIG. 2: (a) The partial electronic density of states (PDOS) of $\mathrm{KGe}_{2}$, (b) the $\mathrm{KGe}_{2}$ band structure, indicating the orbitals with an interlayer nature as filled circles, and (c) the band structure of monolayer germanene. The three plots are aligned at the Fermi level, which is the energy zero.

ing GIC was first realized as a striking "coincidence" in Ref. 4 and is characterized as a hybridized band with significant charge density in the interlayer region. In the case of $\mathrm{KGe}_{2}$, as shown by the small filled circles in Fig. 2(b), there is no occupied band that is dominated by an interlayer nature. Instead, the occupied band in Fig. 2 (b) only has a few momentum points that have an interlayer character as determined by inspecting the wave functions.

Figure 3(a) displays the isotropic Eliashberg spectral function $\alpha^{2} F(\omega)$ for $\mathrm{KGe}_{2} . \alpha^{2} F(\omega)$ displays a large dominant peak centered around $50 \mathrm{meV}$, a second weaker peak centered around $120 \mathrm{meV}$, and a third weaker peak centered at $240 \mathrm{meV}$. The corresponding isotropic electron-phonon coupling strength is $\gamma=1.90$. In order to undestand the vibrational origin of these peaks, we display the atom-projected phonon density of states (PHDOS) in Fig. 3 $3(\mathrm{~b}, \mathrm{c})$. The $\alpha^{2} F(\omega)$ peak centered at $50 \mathrm{meV}$ originates from Ge vibrations, mainly from the Ge out-of-plane modes. The peak centered at 120 meV originate from $\mathrm{K}$ and Ge modes. Regarding $\mathrm{K}$, the main contributing mode is the out-of-plane component, then the planar modes in both the $x$ and $y$ directions. For the Ge contributing modes, again the modes in all directions contribute, where the out-of-plane modes contribute more than the planar modes. Finally, the peak centered at $240 \mathrm{meV}$ does not have any $\mathrm{K}$ contribution. It is driven by Ge $z$ and $y$ modes. That third peak does not have an influence on the value of $\lambda$, as can be seen in the flattening of the $\alpha^{2} F(\omega)$ curve beyond $200 \mathrm{meV}$.

The $\alpha^{2} F(\omega)$ of $\mathrm{KGe}_{2}$ is different to that of $\mathrm{CaC}_{6},{ }^{7}$ where the $\alpha^{2} F(\omega)$ of the latter has three primary peaks,

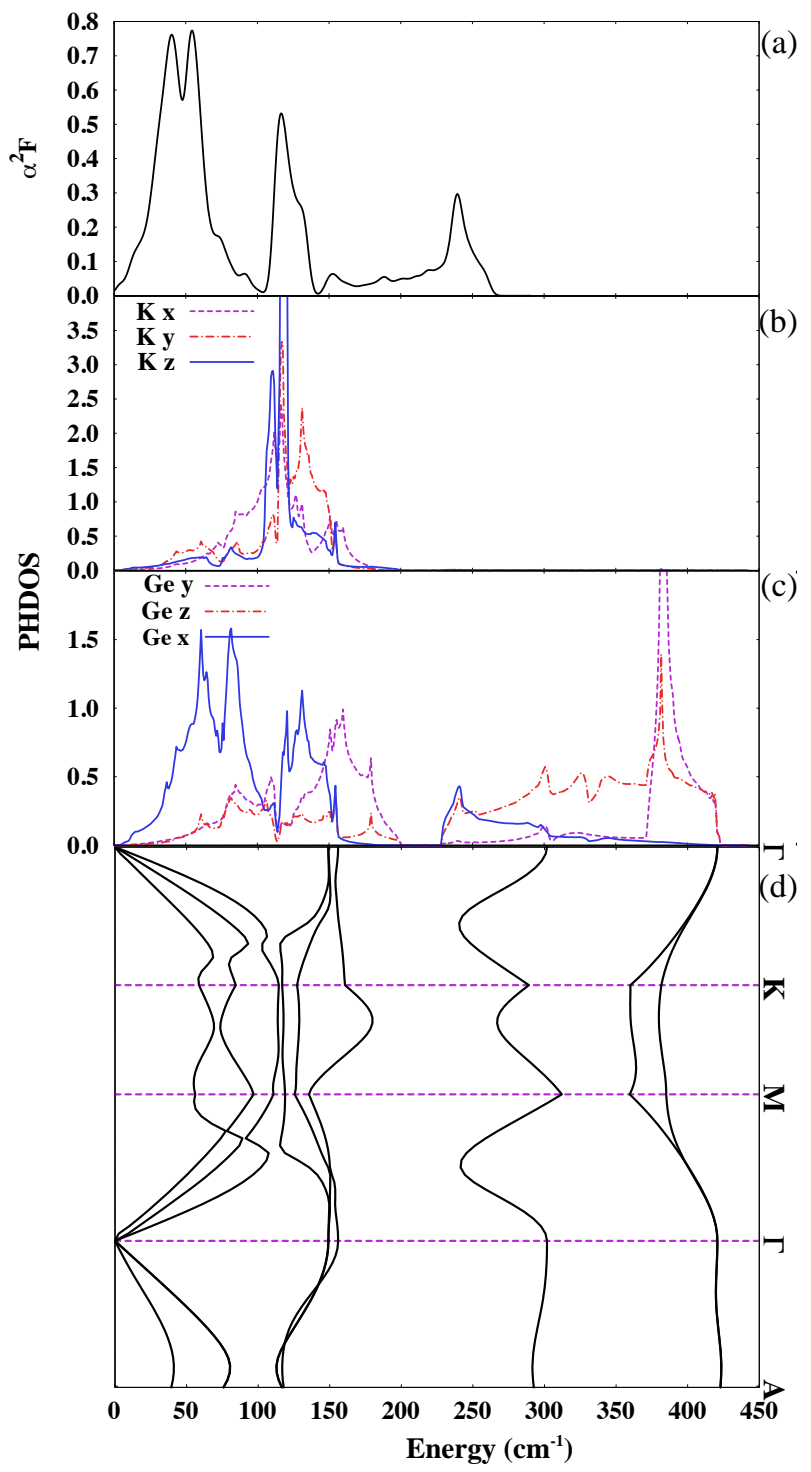

FIG. 3: (a) The Eliashberg function $\alpha^{2} F(\omega)$ of $\mathrm{KGe}_{2}$, the atom-projected phonon density of states (PHDOS) for the (b) $\mathrm{K}$ and the (c) Ge atoms, and the (d) phonon dispersion along the high symmetry points of the Brillouin zone.

the low energy peak being contributed mainly by Ca planar modes, while the second peak is contributed by $\mathrm{C}$ out-of-plane modes, and the third $\mathrm{C}$ peak is contributed by planar modes. First of all, the planar modes in both directions contribute equally in $\mathrm{CaC}_{6}$ due to the lattice symmetry, unlike the case of $\mathrm{KGe}_{2}$ where the positions of the two Ge atoms with respect to the $\mathrm{K}$ atom within unit cell are not symmetric. Second, given that the $\mathrm{K}$ atom is lighter than the Ge atom, the first Ge peak has a lower energy than the $\mathrm{K}$ peak, which is opposite to the case of $\mathrm{CaC}_{6}$, where the first $\mathrm{C}$ peak has a higher energy than the Ca peak. Third, the lowest-energy peak in $\mathrm{CaC}_{6}$ is almost purely Ca dominated, mostly of planar modes, whereas the $\mathrm{K}$ peak has a mixture of $\mathrm{K}$ and $\mathrm{Ge}$ modes, the majority of which are out-of-plane modes. 


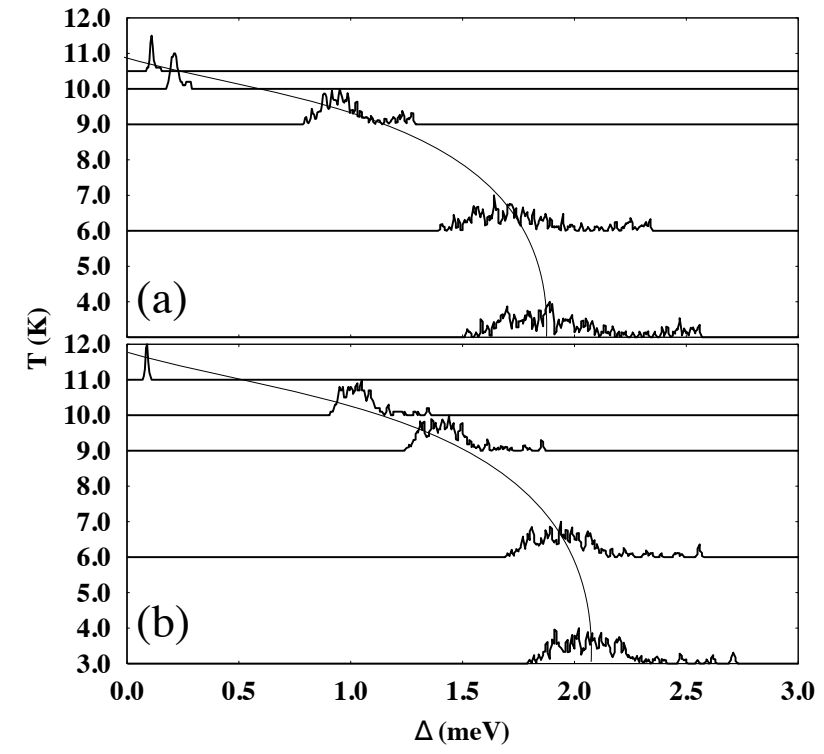

FIG. 4: The superconducting gap function $\Delta(\omega)$ at various values for $T$ for the (a) equilibrium and (b) $5 \%$ tensile-strained $\mathrm{KGe}_{2}$. The curved lines show the trend as the values of $\Delta(\omega)$ converge towards zero.

The calculated electron-phonon coupling is much larger than the values reported for $\mathrm{MgB}_{2}$ and the intercalated graphite compounds, but is closer to the range of values of strong-coupling superconductors such as $\mathrm{Pd}$ We display a list of these values in Tab. I for $2 \mathrm{D}$ intercalated compounds. The reason for this difference is the large buckling of the germanene layer, which leads to the enhanced EPC. The situation in $\mathrm{KGe}_{2}$ is in stark contrast to the $\mathrm{CaGe}_{2}$ compound, where the latter has a very small EPC of 0.19 (cf. Tab I) and does not superconduct.

Within the anisotropic Eliashberg formalism 5 , the value of $T_{c}$ is obtained by examining the gap function $\Delta$ as the temperature parameter $T$ is changed. When $\Delta$ vanishes at some $T$, this characterizes the superconducting state, at which $T_{c}=T$. Such identification is performed by plotting the $\Delta(\omega)$ function at various values of $T$, and inspecting the trend of the function as its peaks converge to zero, as discussed in 5 and displayed in Fig. 4. This figure that the $\Delta(\omega)$ converges to zero as $T$ appraches $T_{c} \sim 11 \mathrm{~K}$. The convergence trend is displayed by a straight line that passes through the various $\Delta(\omega)$ functions.

In the isotropic limit, the Eliashberg formalism reduces to the Allen-Dynes formulation 23 , in which $T_{c}$ is given by

$$
T_{c}=\frac{\omega_{\log }}{1.2} \exp \left[\frac{-1.04(1+\lambda)}{\lambda\left(1-0.62 \mu^{*}\right)-\mu^{*}}\right]
$$

where $\mu^{*}$ is the Coulomb pseudopotential, for which we use $\mu^{*}=0.16$, and the phonon frequencies logarithmic average $\omega_{l o g}$ is given by
TABLE I: The electron-phonon coupling strength $\lambda$, the predicted and experimental superconducting critical temperatures $T_{c}$ for a number of 2D-intercalated compounds.

\begin{tabular}{|c|c|c|c|l|}
\hline Structure & $\lambda$ & $T_{c}$ & $T_{c} \exp$ & \multicolumn{1}{|c|}{ Ref. } \\
\hline $\mathrm{KGe}_{2}$ & 1.9 & 11 & & Present work \\
$\mathrm{MgB}_{2}$ & 0.748 & 50 & 39 & 5 \\
$\mathrm{CaC}_{6}$ & 0.83 & 11 & 11.5 & 7 \\
$\mathrm{CaC}_{2}[95 \mathrm{GPa}]$ & 0.564 & 9.8 & & 24 \\
$\mathrm{LiB}$ & 0.62 & $10-15$ & & 25 \\
\hline
\end{tabular}

$$
\omega_{l o g}=\exp \left[\frac{2}{\lambda} \int \frac{d \omega}{\omega} \alpha^{2} F(\omega) \log \omega\right]
$$

The value of $T_{c}$ calculated for $\mathrm{KGe}_{2}$ using the isotropic Allen-Dynes formalism (Eq. 3) is $5.8 \mathrm{~K}$, which is almost half of the value predicted by solving the full Eliashberg equation. This is because of the significance of the momentum anisotropy in $\mathrm{KGe}_{2}$, which is also the case in $\mathrm{MgB}_{2}$.

Another model for the superconductivity of intercalated compounds is that proposed by Al-Jishi20 for GICs. This is a simplified BCS-based purely electronic model where the graphite $\pi$ and intercalatant $s$ states of are coupled via a coupling parameter. We can easily extend this model to $\mathrm{KGe}_{2}$, where the Hamiltonian couples between the germanene $\pi$ and the K $4 s$ states, and we obtain the equation

$$
k T_{c} \sim \hbar \omega_{c} \exp \left(-\frac{1}{|\lambda|} \sqrt{N_{\pi}(0) N_{4 s}(0)}\right)
$$

where $\omega_{c}$ is the Debye frequency, $N_{\pi}(0)$ and $N_{4 s}(0)$ are the density of states of the $\pi$ and the $\mathrm{K} 4 \mathrm{~s}$ states at the Fermi level, respectively. The critical feature in this equation is that $T_{c}$ would become zero when one of $N_{\pi}(0)$ and $N_{4 s}(0)$ is zero. In our $\mathrm{KGe}_{2}$, full charge transfer occurs from the $\mathrm{K}$ atom to the germanene layer, which should lead to a $T_{c} \sim 0$. The reason is that, with increasing charge gain in the graphite layers in GICs, the $2 \mathrm{D}$ electrons screen the polar coupling between the intercalatant atoms 21 . This, however, is not the case in $\mathrm{KGe}_{2}$, owing to the large electron-phonon coupling contribution arising from the electron-doped germanene layers; that is, superconductivity here is $\pi$-driven (most of $\lambda$ is contributed by the germanene layers), in contrast to the case of GICs where superconductivity is interlayerdriven (most of $\lambda$ is contributed by the Ca atoms) ${ }^{7}$.

\section{CONCLUSIONS}

We predict a superconducting temperature of $\sim 11 \mathrm{~K}$ in a novel buckled intercalated compound, $\mathrm{KGe}_{2}$. The compound has a large electron-phonon coupling of 1.9, 
which decreases by $11 \%$ when a positive planar tensile strain of $5 \%$ is applied. This is acompanied by a slight increase in $T_{c}$ of $\sim 12 \mathrm{~K}$. That is, strong electron-phonon coupling results from the buckled structure of the germanene layers. Despite being an intercalated van der Waals material like intercalated graphite superconductors, $\mathrm{KGe}_{2}$ does not possess an occupied interlayer state.

This research was funded by the Australian Govern- ment through the Australian Research Council (ARC DP160101301). Theoretical calculations were undertaken with resources provided by the National Computational Infrastructure (NCI) supported by the Australian Government and by the Pawsey Supercomputing Centre funded by the Australian Government and the Government of Western Australia.
* Electronic address: sherif.abbas@uts.edu.au

$\dagger$ Electronic address: mike.ford@uts.edu.au

1 Cristina Buzea, and Tsutomu Yamashita, Supercond. Sci. Technol., 2001, 14, 11.

${ }^{2}$ Weller, T. E., Ellerby, M., Saxena, S. S., Smith, R. P. and Skipper, N. T. Nat. Phys. 2005, 1, 3941.

3 K. Sugawara, T. Sato and T. Takahashi, Nat. Physics, 2009, 5, 4043.

4 Gabor Csanyi, P. B. Littlewood, Andriy H. Nevidomskyy, Chris J. Pickard and B. D. Simons, Nature Physics 2005, 1,4245 .

${ }^{5}$ E. R. Margine and F. Giustino, Phys. Rev. B, 2013, 87, 024505.

6 A. M. Fogg, J. B. Claridge, G. R. Darling and M. J. Rosseinsky, Chem. Commun., 2003, 0, 1348-1349.

7 Calandra, M. and Mauri, F. Phys. Rev. Lett. 2005, 95, 237002.

8 A. Acun, L. Zhang, P. Bampoulis, M. Farmanbar, A. van Houselt, A. N. Rudenko, M. Lingenfelder, G. Brocks, B. Poelsema, M. I. Katsnelson and H. J. W. Zandvliet, J. Phys.: Condens. Matter, 2015, 27443002.

9 Jinsong $\mathrm{Xu}$, Jyoti Katoch, Adam S. Ahmed, Igor V. Pinchuk, Justin R. Young, Ezekiel Johnston-Halperin, Jonathan Pelz, Roland K.Kawakami, J. Cryst. Gr., 2017, 460, 134-138.

10 Tianping Ying, Xiaolong Chen, Gang Wang, Shifeng Jin, Xiaofang Lai, Tingting Zhou, Han Zhang, Shijie Shen, and Wanyan Wang, J. Am. Chem. Soc., 2013, 135, 2951-2954.

11 R. B. Somoano, V. Hadek, and A. Rembaum, J. Chem. Phys., 1973, 58, 2.
12 D. M. Ceperley and B. J. Alder, Phys. Rev. Lett., 1980, 45,566 .

13 N. Troullier and J. L. Martins, Phys. Rev. B, 1991, 43, 1993.

14 P. Giannozzi et al., J. Phys. Condens. Matter, 2009, 21, 395502.

15 M. Methfessel and A. T. Paxton, Phys. Rev. B, 1989, 40, 3616.

16 S. Baroni, S. de Gironcoli, A. Dal Corso, and P. Giannozzi, Rev. Mod. Phys., 2001, 73, 515.

17 N. Kolmogorov and S. Curtarolo, Phys. Rev. B, 2006, 73, 180501R.

18 Sudipta Dutta and Katsunori Wakabayashi, Phys. Rev. B, 2015, 91, 201410R.

19 Electronic structure and electron-phonon coupling of doped graphene layers in KC8, Phys. Rev. B, 2009, 79, 205106.

20 R. Al-Jishi, Phys. Rev. B, 1983, 28, 1.

21 Yasutami Takada, Journal of the Physical Society of Japan, 2009, 78, 1.

22 L. Liu, Journal of Physics and Chemistry of Solids, 1986, 47, 1067-1072.

23 P. B. Allen and R. C. Dynes, Phys. Rev., 1975, B 12, 905.

24 Yan-Ling Lia,b, Wei Luob,c, Zhi Zengd, Hai-Qing Line, Ho-kwang Maof,g,1, and Rajeev Ahuja, Proc. Nat. Acad. Sci., 2013, 110, 9289-9294.

25 Matteo Calandra, Aleksey N. Kolmogorov and Stefano Curtarolo, Phys. Rev. B, 2007, 75, 144506. 